Pacific Journal of Mathematics

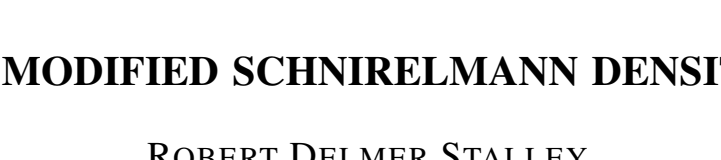




\title{
A MODIFIED SCHNIRELMANN DENSITY
}

\author{
ROBERT STALLEY
}

1. Introduction. We define a modified Schnirelmann density for an infinite sequence of positive integers and prove two theorems about this density which are analogous but not identical to well-known results for Schnirelmann density.

Henceforth we assume all sequences are infinite. Let $A$ be a sequence of positive integers $a_{1}<a_{2}<\ldots$, let $A(n)$ be the number of integers of $A$ not greater than $n$, and let $l$ be the sequence of all positive integers. Then the wellknown asymptotic density $\delta(A)$, the Schnirelmann density $\alpha$, and the modified Besicovitch density $a_{1}$, of $A$, are defined as follows:

$$
\begin{aligned}
\delta(A) & =\lim \inf \frac{A(n)}{n} ; \\
\alpha & =\operatorname{glb} \frac{A(n)}{n} ; \\
a_{1} & =\underset{n \geq s}{\operatorname{glb}} \frac{A(n)}{n+1},
\end{aligned}
$$

where $A \neq I$ and $s$ is the smallest positive integer missing from $A$. We define the modified Schnirelmann density or more briefly the modified density $a^{*}$ of $A$ as follows:

$$
\alpha^{*}=\operatorname{glb} \frac{i}{a_{i}}
$$

Thus the modified density may be defined by merely restricting to $A$ the $n$ occurring in the definition of Schnirelmann density.

Let $B$ be the sequence of positive integers $b_{1}<b_{2}<\ldots$. The sum $C=A+B$ of the sequences $A$ and $B$ is defined as the sequence of integers of the form

Received May 29, 1953. Results in this paper were included in a doctoral dissertation written under the direction of Professor Ivan Niven at the University of Oregon, 1953.

Pacific J. Math. 5 (1955), 119-124 
$a_{i}$ or $b_{i}$ or $a_{i}+b_{j}$. The well-known $\alpha+\beta$ theorem $[1 ; 3]$ states the following:

$$
\begin{aligned}
& \text { if } \alpha+\beta \geq 1 \text {, then } \gamma=1 \text {; } \\
& \text { if } \alpha+\beta<1 \text {, then } \gamma \geq \alpha+\beta \text {. }
\end{aligned}
$$

That neither part of this theorem holds with Schnirelmann density replaced by modified density is shown by the examples $A=B=\{1,3+j\} \quad(j=1,2, \ldots)$, and $A=B=\{1,4+j\} \quad(j=1,2, \ldots)$, respectively. Theorems 1 and 2 for modified density are analogous to $(1)$ and $(2)$, respectively.

\section{The analogue of the $\alpha+\beta$ theorem.}

THEOREM 1. If $\alpha^{*}+\beta^{*}>1$, then $\gamma^{*}=1$.

Proof. We must prove $C=I$. Suppose $n$ is missing, let $n+t$ and $n+u$ be the next larger members of $A$ and $B$ respectively, and let $A(n)=r$. First,

$$
\alpha^{*} \leq \frac{A(n+t)}{n+t}=\frac{r+1}{n+t} \leq \frac{r+1}{n+1} .
$$

Next, $B$ lacks at least the integers $n-a_{r}, n-a_{r-1}, \cdots, n-a_{1}, n, \cdots, n+u-1$, and so

$$
\beta^{*} \leq \frac{B(n+u)}{n+u} \leq \frac{n-r}{n+u} \leq \frac{n-r}{n+1} .
$$

Therefore

$$
\alpha^{*}+\beta^{*} \leq \frac{r+1}{n+1}+\frac{n-r}{n+1}=1
$$

a contradiction, and the theorem is proved.

Simple examples show that (1) and Theorem 1 give independent conditions for $C=I$.

THEOREM 2. If $\alpha^{*}+\beta^{*} \leq 1$ and $1,2, \ldots, k \in A$, then

$$
\gamma^{*} \geq \frac{k}{k+1} \alpha^{*}+\beta^{*}
$$

Theorem 2 is a best possible result in the sense that there exist sequences $A$ and $B$ such that equality holds and such that $0<\alpha^{*}<1$ and $0<\beta^{*}<1$. 
This is shown by the following example.

EX AM PLE 1. $A=B=\{1,2, \ldots, k, 4 k+j\}$ where $j=0,1,2, \ldots$

Example 1 together with Example 2 show that the hypothesis $1,2, \ldots, k \in A$ of Theorem 2 is necessary.

ExAMPLE 2. $A=\{s+j\}$ and $B=\{1,2, \cdots, r-1, s+j\}$, where $j=0,1,2, \ldots$ and $1 \leq r \leq s$.

In order to prove Theorem 2 we first prove Lemmas 1 and 2. Lemma 1 depends on the following result of H. B. Mann [4]: If $n_{i}$ is the $i$ th positive integer missing from $C$, and $n$ is an arbitracy positive integer missing from $C$, then

$$
C(n) \geq \alpha_{1} n+B(n)+\min _{n_{i} \leq n}\left\{A\left(n_{i}\right)-\alpha_{1} n_{i}\right\}
$$

Lemma 1. If $\alpha^{*}+\beta^{*} \leq 1$ and $A \neq l$, then $\gamma^{*} \geq \alpha_{1}+\beta^{*}$.

Proof. If $C=I$, then by hypothesis

$$
\gamma^{*}=1 \geq \alpha^{*}+\beta^{*} \geq \alpha_{1}+\beta^{*},
$$

and we are through.

If $C \neq I$, we restrict $n$ so that $n \notin C$ but $(n+1) \in C$. For $n$ fixed, (3) yields

$$
C(n) \geq \alpha_{1} n+B(n)+A\left(n_{d}\right)-\alpha_{1} n_{d}
$$

for fixed $n_{d} \notin C$. By definition, $\alpha_{1} \leq A\left(n_{d}\right) /\left(n_{d}+1\right)$, or equivalently

$$
\alpha_{1} n+A\left(n_{d}\right)-\alpha_{1} n_{d} \geq \alpha_{1}(n+1),
$$

and so (4) may be written

$$
C(n) \geq \alpha_{1}(n+1)+B(n) .
$$

Therefore

$$
C(n+1)=C(n)+1 \geq \alpha_{1}(n+1)+B(n)+1=\alpha_{1}(n+1)+B(n+u)
$$

where $n+u$ is the smallest member of $B$ greater than $n$. Hence

$$
C(n+1) \geq \alpha_{1}(n+1)+\beta^{*}(n+u) \geq \alpha_{1}(n+1)+\beta^{*}(n+1),
$$

and division by $n+1$ yields 


$$
\frac{C(n+1)}{n+1} \geq \alpha_{1}+\beta^{*}
$$

Finally, (5) yields

$$
\gamma^{*}=\operatorname{glb} \frac{C(n+1)}{n+1} \geq \alpha_{1}+\beta^{*}
$$

and Lemma 1 is proved.

Lемма 2. If $A \neq I$ and $1,2, \cdots, k \in A$, then

$$
\alpha_{1} \geq \frac{k}{k+1} \alpha^{*}
$$

Proof. We restrict $n$ so that $n \notin A$ but $n+1 \in A$. Clearly

$$
\alpha^{*}=\operatorname{glb} \frac{A(n+1)}{n+1} \text { and } \alpha_{1}=\operatorname{glb} \frac{A(n)}{n+1} \text {. }
$$

Hence

$$
\begin{aligned}
\alpha_{1} & =\operatorname{glb} \frac{A(n)}{n+1}=\operatorname{glb}\left\{\frac{A(n)}{A(n+1)} \frac{A(n+1)}{n+1}\right\} \\
& \geq \operatorname{glb} \frac{A(n)}{A(n+1)} \operatorname{glb} \frac{A(n+1)}{n+1} \geq \frac{k}{k+1} \alpha^{*} .
\end{aligned}
$$

Proof of Theorem 2. If $C=I$, then by hypothesis

$$
\gamma^{*}=1 \geq \alpha^{*}+\beta^{*} \geq \frac{k}{k+1} \alpha^{*}+\beta^{*},
$$

and we are through. If $C \neq I$ then $A \neq I$, and so Lemmas 1 and 2 yield

$$
\gamma^{*} \geq \alpha_{1}+\beta^{*} \geq \frac{k}{k+1} \alpha^{*}+\beta^{*}
$$

This completes the proof of Theorem 2.

It is striking that while these results for modified density differ considerably from the analogous results for Schnirelmann density, they differ very little from the following results for asymptotic density due to H. -H. Ostmann [5]: 


$$
\begin{aligned}
& \text { If } \delta(A)+\delta(B)>1 \text {, then } \delta(C)=1 . \\
& \begin{array}{r}
\text { If } \delta(A)+\delta(B) \leq 1 \text { and } x, x+1, \cdots, x+k \in A \cup 0, \\
\text { then } \delta(C) \geq \frac{k}{k+1} \delta(A)+\delta(B) .
\end{array}
\end{aligned}
$$

If asymptotic density is replaced by modified density, (6) becomes Theorem 1 , and (7) for $x=0$ becomes Theorem 2 .

3. The density of a sum. If the hypothesis $1,2, \cdots, k \in A$ of Theorem 2 is completely removed, then, according to Example 2, $\gamma^{*} \geq \max \left\{\alpha^{*}, \beta^{*}\right\}$ is the strongest inequality obtainable for the density of $C$. However, the question arises as to the existence of a stronger inequality when this hypothesis of Theorem 2 is replaced by some different assumption. The following theorem is an example of such a result.

THEOREM 3. If $\gamma^{*}<i / c_{i}$ for all $i>0$, and there are $k$ consecutive integers in $A$, then

$$
\gamma^{*} \geq \frac{k-1}{k} \alpha^{*}+\beta^{*}
$$

Proof. First we note that $\gamma^{*}=\lim \inf i / c_{i}$ and hence $\gamma^{*}=\delta(C)$. Next since $\gamma^{*}<1$ then $\delta(C)<1$, and so $\delta(A)+\delta(B) \leq 1$ by $(6)$. Therefore (7) yields

$$
\gamma^{*}=\delta(C) \geq \frac{k-1}{k} \delta(A)+\delta(B) \geq \frac{k-1}{k} \alpha^{*}+\beta^{*},
$$

and the theorem is proved.

Further light is shed on the relation of $\gamma^{*}$ to $\alpha^{*}$ and $\beta^{*}$ by use of a result of L. P.-H. Cheo [2]. He showed that for given nonnegative values of $\alpha, \beta$, and $\gamma$ satisfying $1 \geq \gamma \geq \alpha+\beta$ there exist sequences $A, B$, and $C=A+B$ having Schnirelmann densities $\alpha, \beta$, and $\gamma$, respectively. Cheo's proof is constructive, and for his sequences $\alpha^{*}=\alpha, \beta^{*}=\beta$, and $\gamma^{*}=\gamma$. Therefore Cheo's result holds for modified density. The interval of possible values for $\gamma^{*}$ cannot be lengthened to the larger interval $\left[k(k+1)^{-1} \alpha^{*}+\beta^{*}, 1\right]$, as suggested by Theorem 2, for we cannot have

$$
\gamma^{*}=k(k+1)^{-1} \alpha^{*}+\beta^{*}
$$

when $\beta^{*}=0$ and $\alpha^{*}>0$. 
Finally, if the modified density of a finite sequence is defined to be zero, then all results and discussions in this paper clearly hold without the restriction that the sequences be infinite.

\section{REFERENCES}

1. E. Artin, and P. Scherk, On the sum of two sets of integers, Ann. of Math. 44 (1943), $138-142$.

2. L. P.-H. Cheo, A remark on the $\alpha+\beta$ theorem, Proc. Amer. Math. Soc. 3 (1952), $175-177$.

3. H. B. Mann, A proof of the fundamental theorem on the density of sums of sets of positive integers, Ann. of Math. 43 (1942), 523 - 527.

4. - On the number of integers in the sum of two sets of positive integers, Pacific J. Math. 1 (1951), 249-253.

5. H. -H. Ostmann, Verfeinerte Lösung der asymptotischen Dichtenaufgabe, J. Reine Angew. Math. 187 (1950), $183-188$.

IOWA STATE COLLEGE 



\section{Pacific Journal of Mathematics}

\section{Vol. 5, No. $1 \quad$ September, 1955}

Frank Herbert Brownell, III, Flows and noncommuting projections on

Hilbert space ................................... 1

H. E. Chrestenson, A class of generalized Walsh functions ............. 17

Jean Bronfenbrenner Crockett and Herman Chernoff, Gradient methods of maximization ................................... 33

Nathan Jacob Fine, On groups of orthonormal functions. I .......... 51

Nathan Jacob Fine, On groups of orthonormal functions. II ............ 61

Frederick William Gehring, A note on a paper by L. C. Young .......... 67

Joachim Lambek and Leo Moser, On the distribution of Pythagorean

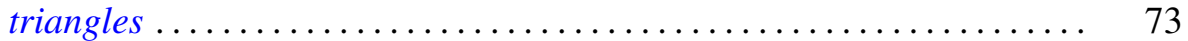

Roy Edwin Wild, On the number of primitive Pythagorean triangles with

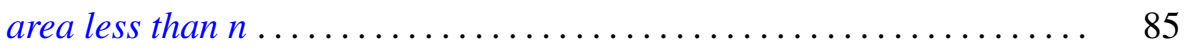

R. Sherman Lehman, Approximation of improper integrals by sums over multiples of irrational numbers ........................ 93

Emma Lehmer, On the number of solutions of $u^{k}+D \equiv w^{2}(\bmod p) \ldots 103$

Robert Delmer Stalley, A modified Schnirelmann density............... 119

Richard Allan Moore, The behavior of solutions of a linear differential equation of second order............................. 125

William M. Whyburn, A nonlinear boundary value problem for second order differential systems. 